\title{
Nearly universal crossing point of the specific heat curves of Hubbard models
}

\author{
N. Chandra,* M. Kollar, and D. Vollhardt \\ Theoretische Physik III, Elektronische Korrelationen und Magnetismus, Institut für Physik, \\ Universität Augsburg, 86135 Augsburg, Germany
}

(Received 28 October 1998)

\begin{abstract}
A nearly universal feature of the specific heat curves $C(T, U)$ vs $T$ for different $U$ of a general class of Hubbard models is observed. That is, the value $C_{+}$of the specific heat curves at their high-temperature crossing point $T_{+}$is almost independent of lattice structure and spatial dimension $d$, with $C_{+} / k_{B} \approx 0.34$. This surprising feature is explained within second-order perturbation theory in $U$ by identifying two small parameters controlling the value of $C_{+}$: the integral over the deviation of the density of states $N(\epsilon)$ from a constant value, characterized by $\delta N=\int d \epsilon\left|N(\epsilon)-\frac{1}{2}\right|$; and the inverse dimension $1 / d$. [S0163-1829(99)15015-X]
\end{abstract}

\section{INTRODUCTION}

Recently attention was drawn to the fact that in various strongly correlated systems the curves of the specific heat $C(T, X)$ vs temperature $T$ cross once or twice when plotted for different values of a second thermodynamic variable $X$. ${ }^{1}$ For example, crossing points are observed for different pressures $(X=P)$ in normalfluid ${ }^{3} \mathrm{He}$ (Ref. 2) and heavy-fermion systems such as $\mathrm{CeAl}_{3}$ (Ref. 3) and $\mathrm{UBe}_{3} .{ }^{4}$ By changing the magnetic field $(X=B)$, the same feature is seen in heavyfermion compounds such as $\mathrm{CeCu}_{6-x} \mathrm{Al}_{x}$ (Ref. 5) and $\mathrm{Nd}_{2-x} \mathrm{Ce}_{x} \mathrm{CuO}_{4}{ }^{6}$ Crossings of the specific heat curves are also observed in the simplest lattice model for correlated electrons, the Hubbard model, ${ }^{7}$

$$
\hat{H}=\sum_{\mathbf{k} \sigma}\left(\epsilon_{\mathbf{k}}-\mu\right) \hat{a}_{\mathbf{k} \sigma}^{+} \hat{a}_{\mathbf{k} \sigma}+U \sum_{i} \hat{n}_{i \uparrow} \hat{n}_{i \downarrow},
$$

where $\epsilon_{\mathbf{k}}$ is the dispersion of a single electronic band, $\mu$ the chemical potential, and $U$ the local interaction. At half filling the curves $C(T, U)$ vs $T$ always cross at two temperatures. This is observed, for example, in the case of the model with nearest-neighbor hopping in $d=1,8,9 d=2,{ }^{10}$ and $d=\infty,{ }^{11}$ as well as for long-range hopping in $d=1$ (Ref. 12); for the latter two systems the specific heat is shown in Fig. 1. Furthermore, crossing is found in $d=1$ when a magnetic field $B$ is changed at constant $U .{ }^{13}$ The fact that these crossing points may be very sharp was analyzed in Ref. 1, and was traced to the properties of certain generalized susceptibilities of the system.

In the following, we will consider only the crossing of the specific-heat curves occurring for $X=U$ in the paramagnetic phase of the Hubbard model with a symmetric half-filled band $(n=1)$. We will investigate yet another observation, namely, that for small $U$ the specific heat at the hightemperature crossing point has practically the same value of approximately $0.34 k_{B}$ for all dimensions $d$ and dispersions $\epsilon_{\mathrm{k}}$, which can be seen also in Fig. 1. This is surprising, because the temperatures at which this crossing occurs are very different for different dispersions and dimensions, and because the maximum value of $C(T, U)$ and its value at the low-temperature crossing point vary strongly as well. It should be noted that the specific heat, like the entropy, is a dimensionless quantity when expressed in units of $k_{B}$.

We denote by $T_{+}$the temperature at which the curves $C(T, U)$ vs $T$ cross for different values of $U$. Then the specific heat is independent of $U$ at the crossing temperature $T_{+}(U)$, defined by ${ }^{1}$

$$
\left.\frac{\partial C}{\partial U}\right|_{T_{+}(U)}=0 .
$$

Since we are not concerned with the dependence of $T_{+}(U)$ on $U$, but rather with the crossing point value of $C(T, U)$ for different lattice systems, we consider only the limit of small $U$, and define

$$
\begin{gathered}
T_{+} \equiv \lim _{U \rightarrow 0^{+}} T_{+}(U), \\
C_{+} \equiv \lim _{U \rightarrow 0^{+}} C\left[T_{+}(U), U\right] .
\end{gathered}
$$

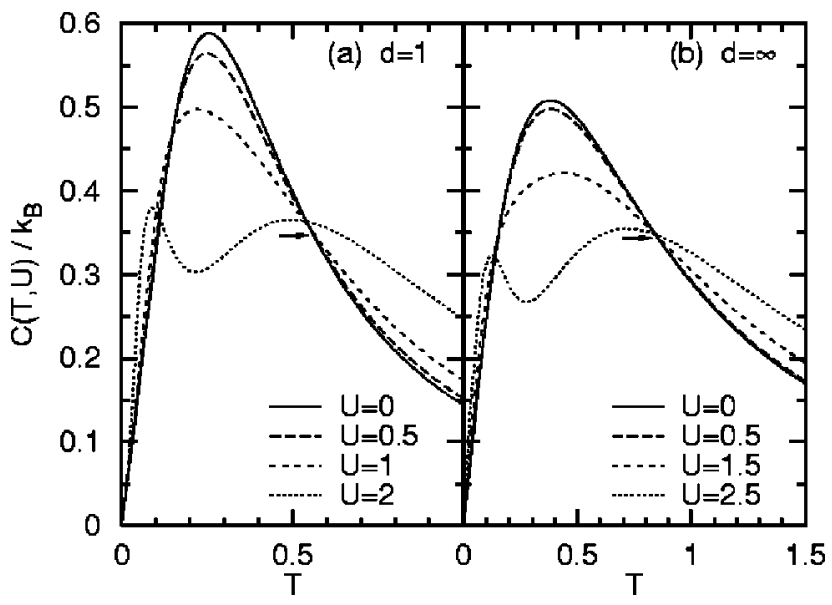

FIG. 1. Specific heat for the Hubbard model. (a) Exact solution for $1 / r$ hopping in $d=1 .^{12}$ (b) Iterated perturbation theory for NN hopping in $d=\infty$ (Ref. 11). In (a), $T$ and $U$ are in units of the half-band-width, while for (b) the second moment of the (Gaussian) density of states is set to unity. At the high-temperature crossing point the specific heat has the almost universal value of $0.34 k_{B}$ in the limit $U \rightarrow 0$ (see arrows). 
This weak-coupling crossing point can be calculated without approximation using second-order perturbation theory in $U$. Higher orders in perturbation theory would be necessary to determine the dependence of $C\left[T_{+}(U), U\right]$ on $U$.

It is the purpose of this paper to show that the value of $C_{+}$at the high-temperature crossing point is almost universal, and to analyze the origin of this peculiar feature. We calculate $C_{+}$for a half-filled band with a symmetric density of states (DOS) $N(\boldsymbol{\epsilon})=\int d \mathbf{k} \delta\left(\epsilon-\epsilon_{\mathbf{k}}\right)$. We also show that the weak dependence of $C_{+}$on lattice properties can be understood by starting from the limit $d=\infty$ and using expansions in terms of two small parameters.

This paper is organized as follows. In Sec. II we review the origin of crossing points in the Hubbard model and show how to calculate $T_{+}$and $C_{+}$in second-order perturbation theory, the details of which are contained in the Appendix. Various noninteracting lattice systems are listed in Sec. III, and the values of $C_{+}$at the high-temperature crossing point for these systems are presented in Sec. IV. Section V contains expansions of $C_{+}$that reveal the influence of the density of states and the lattice dimension. We close with a conclusion in Sec. VI.

\section{CROSSING POINTS IN THE SPECIFIC HEAT OF THE HUBBARD MODEL}

The entropy per lattice site $S(T, U)$ is given by

$$
S(T, U)=\int_{0}^{T} d T^{\prime} \frac{C\left(T^{\prime}, U\right)}{T^{\prime}} .
$$

For the Hubbard model, $S(T, U)$ approaches a constant when $T \rightarrow \infty$. Taking the derivative of Eq. (5) with respect to $U$, we find

$$
0=\int_{0}^{\infty} \frac{d T}{T} \frac{\partial C(T, U)}{\partial U}
$$

Since $\partial C / \partial U$ is not identically zero, there must exist temperature regions where it has positive and negative values. We assume that no phase transitions occur, so that $\partial C / \partial U$ is a continuous function of $U$. Then there exist temperatures where $\partial C / \partial U$ changes sign; at these temperatures the curves $C(T, U)$ vs $T$ cross [see Eq. (2)].

There are two such crossing points in the paramagnetic phase of the half-filled Hubbard model, as can be seen from the sign of $\partial C / \partial U$ at very low and very high temperatures. For intermediate values of $U$ the specific heat of this model shows the following general features: $C(T, U)$ starts linearly in $T$ at low temperatures and develops a two-peak structure; one at temperatures $T \sim 4 t^{2} / U$ due to spin excitations ( $t$ is the hopping amplitude); and one at temperatures $T \sim U-W$ due to charge excitations, where $W$ is the bandwidth. For low temperatures the spin exitations become stronger for increasing $U$, thus $\partial C / \partial U>0$. At high temperatures an increase in $U$ pushes out the charge peak and thus increases $C(T, U)$, which tends to $\left[a+b U^{2}+O\left(U^{4}\right)\right] / T^{2}$ with $a, b>0$. Hence $\partial C / \partial U>0$ for both high and low temperatures, so that the sum rule [Eq. (6)] yields an intermediate region with $\partial C / \partial U<0$. There are thus two sign changes corresponding to two crossing points.

To determine the location of the crossing points defined by Eqs. (3) and (4), we calculate the internal energy per lattice site in perturbation theory in $U$,

$$
E(T, U)=E^{(0)}(T)+\frac{1}{4} U+U^{2} E^{(2)}(T)+O\left(U^{3}\right) .
$$

Here $\left(k_{B} \equiv 1, \beta=1 / T\right)$

$$
E^{(0)}(T)=2 \int d \epsilon \frac{N(\epsilon) \epsilon}{1+\exp (\beta \epsilon)},
$$

which is the internal energy for the noninteracting system, $U / 4$ is the Hartree contribution, and the second-order correlation energy is given by (see the Appendix for details)

$$
E^{(2)}(T)=-\frac{\partial}{\partial \beta} \frac{\beta^{2}}{32} \int_{0}^{1} d x \int d \mathbf{k} \int d \mathbf{p} \int d \mathbf{q} \frac{\cosh \left[\frac{1}{2} x \beta\left(\epsilon_{\mathbf{k}}+\epsilon_{\mathbf{p}}+\epsilon_{\mathbf{p}+\mathbf{q}}+\epsilon_{\mathbf{k}+\mathbf{q}}\right)\right]}{\cosh \left(\frac{1}{2} \beta \epsilon_{\mathbf{k}}\right) \cosh \left(\frac{1}{2} \beta \epsilon_{\mathbf{p}}\right) \cosh \left(\frac{1}{2} \beta \epsilon_{\mathbf{k}+\mathbf{q}}\right) \cosh \left(\frac{1}{2} \beta \epsilon_{\mathbf{p}+\mathbf{q}}\right)},
$$

where the integrations, e.g., $\int d \mathbf{k} \equiv \int d^{d} k /(2 \pi)^{d}$, run over the first Brillouin zone.

In the limit of infinite spatial dimensions ${ }^{14}$ this expression can be simplified further. In this case, momentum conservation at vertices becomes irrelevant, ${ }^{15}$ so that the integrals factorize (see the Appendix):

$$
E^{(2)}(T)=-\frac{\partial}{\partial \beta} \frac{\beta^{2}}{32} \int_{0}^{1} d x\left[\int d \epsilon N(\epsilon) \frac{\cosh \left(\frac{1}{2} x \beta \epsilon\right)}{\cosh \left(\frac{1}{2} \beta \epsilon\right)}\right]^{4}
$$

Note that as usual in infinite dimensions the dispersion $\epsilon_{\mathbf{k}}$ enters into one-particle quantities only via the DOS $N(\epsilon)$. Therefore this expression is much easier to evaluate numerically than Eq. (9).
The specific heat $C(T, U)=\partial E / \partial T$ has the expansion

$$
C(T, U)=C^{(0)}(T)+U^{2} C^{(2)}(T)+O\left(U^{4}\right),
$$

where

$$
C^{(0)}(T)=\frac{\beta^{2}}{2} \int d \epsilon \frac{N(\epsilon) \epsilon^{2}}{\cosh ^{2}\left(\frac{1}{2} \beta \epsilon\right)},
$$

and the function $C^{(2)}(T)$ can be written as (see the Appendix)

$$
C^{(2)}(T)=\frac{\beta^{2}}{32} \frac{\partial^{2}}{\partial \beta^{2}} \beta^{2} \int_{0}^{1} d x \sum_{m}\left[f_{m}(x, \beta)\right]^{4} .
$$


Here the sum runs over lattice sites $\mathbf{R}_{m}$, and the functions $f_{m}(x, \beta)$ are given by

$$
f_{m}(x, \beta)=\int d \mathbf{k} \frac{\exp \left(i \mathbf{k} \cdot \mathbf{R}_{m}+\frac{1}{2} x \beta \epsilon_{\mathbf{k}}\right)}{\cosh \left(\frac{1}{2} \beta \epsilon_{\mathbf{k}}\right)} .
$$

Comparison with Eq. (10) shows that in $d=\infty$ only the local term with $\quad \mathbf{R}_{m}=0$, i.e. $f_{0}(x, \beta)=\int d \epsilon N(\epsilon) \cosh \left(\frac{1}{2} x \beta \epsilon\right) /$ $\cosh \left(\frac{1}{2} \beta \epsilon\right)$, contributes to the sum in Eq. (13).

The crossing point in the specific heat occurs at the temperature $T_{+}(U)$ for which $C(T, U)$ is independent of $U$. In view of Eqs. (2), (3), and (11), the crossing temperature $T_{+}$ in the limit $U \rightarrow 0$ is given by the root of the equation

$$
C^{(2)}\left(T_{+}\right)=0,
$$

and the specific heat at the crossing point $[\mathrm{Eq}$. (4)] is

$$
C_{+}=C^{(0)}\left(T_{+}\right) .
$$

These equations will be evaluated for several lattices and dimensions that are described in Sec. III.

\section{MOMENTUM DISPERSION AND DENSITY OF STATES}

We consider only one-band systems at half-filling with a symmetric density of states on lattices in finite and infinite spatial dimensions. For systems with finite bandwidth we set $W / 2 \equiv 1$, where $W$ is the bandwidth, while for infinite bandwidth we use a unit second moment of the density of states, i.e., $\int d \epsilon N(\epsilon) \epsilon^{2} \equiv 1$.

(1) Finite dimensions. For the linear chain, square lattice, and simple cubic lattice, i.e., the hypercubic lattices in $d$ $=1,2$, and 3 , we use the tight-binding dispersion $\epsilon_{\mathbf{k}}$ $=-2 t \sum_{i=1}^{d} \cos k_{i}, \quad\left|k_{i}\right| \leqslant \pi$, which describes nearestneighbor (NN) hopping with amplitude $t \equiv 1 / 2 d$. Furthermore we study the body-centered-cubic (bcc) lattice in $d=3$ with NN hopping, which can be regarded as a subset of the simple cubic lattice with hopping across the space-diagonal, so that $\epsilon_{\mathbf{k}}=-8 t \cos \left(k_{x}\right) \cos \left(k_{y}\right) \cos \left(k_{z}\right)$ with $t \equiv \frac{1}{8}$. For these systems we use perturbation theory as described in Sec. II. Finally, for one-dimensional long-range hopping $t(r) \propto 1 / r$, the known interacting dispersion ${ }^{12}$ can be used instead of perturbation theory. The free dispersion is $\epsilon_{k}=t k, t \equiv 1 / \pi$, with a constant density of states. Figure 2 shows the various densities of states used in $d=1,2$, and 3 .

(2) Infinite dimensions. We consider first the hypercubic lattice and generalized honeycomb lattice with NN hopping. For the hypercubic lattice, the hopping must be scaled ${ }^{14}$ as $t=1 / \sqrt{2 d}$ to obtain a nontrivial limit for $d \rightarrow \infty$. In this case the density of states becomes a Gaussian with unit variance, $N(\epsilon)=\exp \left(-\epsilon^{2} / 2\right) / \sqrt{2 \pi}$, whereas for the generalized honeycomb lattice ${ }^{16}$ the same scaling leads to $N(\epsilon)=|\epsilon| \exp \left(-\epsilon^{2}\right)$. We also study the Bethe lattice with infinite connectivity and semicircular density of states, $N(\epsilon)=2 / \pi \sqrt{1-\epsilon^{2}},|\epsilon| \leqslant 1$.

Furthermore we can take advantage of the fact that in $d$ $=\infty$ only the density of states of the non-interacting system appears in Eq. (10). Hence it may be chosen at will even when no corresponding dispersion is known. We consider three such functions each containing a tunable real parameter

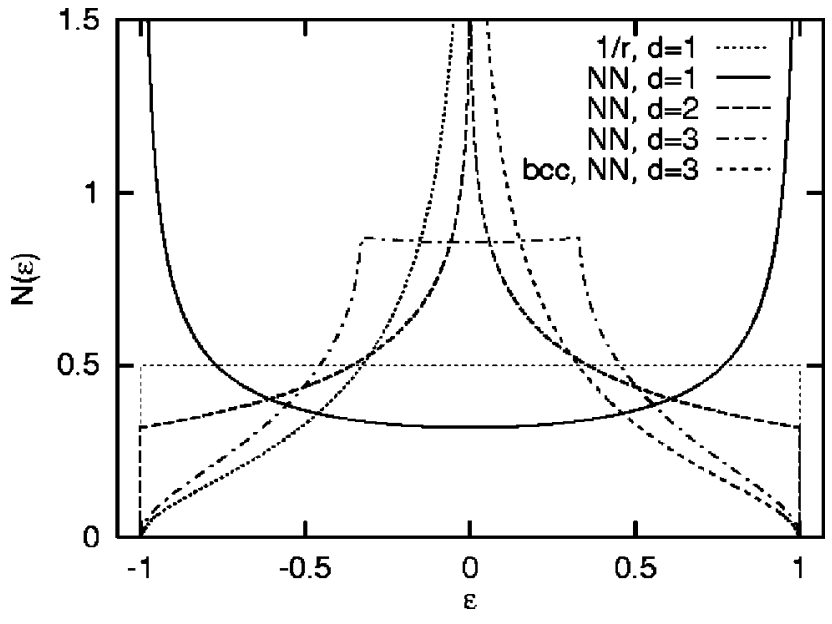

FIG. 2. DOS's for several noninteracting systems with nextneighbor and $1 / r$ hopping in dimensions $d=1,2$, and 3 .

$\alpha>0$. This allows us to study the behavior of $C_{+}$for a wide range of DOS shapes. The first is the "metallic" density of states

$$
N(\epsilon)=\frac{1+\alpha}{2 \alpha}\left(1-|\epsilon|^{\alpha}\right), \quad|\epsilon| \leqslant 1,
$$

bearing this name due to its finite value at the Fermi energy, while the "semimetallic" density of states

$$
N(\epsilon)=\frac{1+\alpha}{2}|\epsilon|^{\alpha}, \quad|\epsilon| \leqslant 1,
$$

vanishes at the Fermi energy. Furthermore we employ a semimetallic density of states "with tails" having infinite bandwidth and unit variance

$$
N(\epsilon)=\frac{c^{c}}{\Gamma(c)}|\epsilon|^{\alpha} \exp \left(-c \epsilon^{2}\right), \quad c \equiv \frac{1+\alpha}{2} .
$$

This reduces to the DOS for the generalized honeycomb lattice with NN hopping in the case of $\alpha=1$. The important special case of a constant rectangular density of states, $N(\epsilon)=\frac{1}{2}$ for $|\epsilon| \leqslant 1$, is contained in Eq. (17) for $\alpha \rightarrow \infty$ and in Eq. (18) for $\alpha \rightarrow 0$. Note that in the limit $\alpha \rightarrow \infty$, the DOS in Eq. (18) approaches two $\delta$ peaks, $N(\epsilon)=\frac{1}{2}[\delta(1+\epsilon)$ $+\delta(1-\epsilon)]$. This particular case is of interest only because in this case the integrals in Eqs. (8) and (10) can be calculated analytically. For general $\alpha$, on the other hand, the functions in Eqs. (17) and (18) model typical DOS shapes for finite dimensions. Several densities of states used in $d=\infty$ are depicted in Fig. 3.

\section{RESULTS FOR THE SPECIFIC HEAT AT THE HIGH-TEMPERATURE CROSSING POINT}

In this section we present results for the specific heat $C_{+}$ at the high-temperature crossing point for the density of states discussed in Sec. III. To calculate $C_{+}$according to Eqs. (15) and (16), the integrals appearing in Eqs. (12) and (13) have to be calculated numerically. We determine them to high precision (typically $10^{-8}$ ) by either Monte Carlo integration (using the VEGAS algorithm ${ }^{17}$ ) or by a hightemperature expansion (described in the Appendix). For sev- 


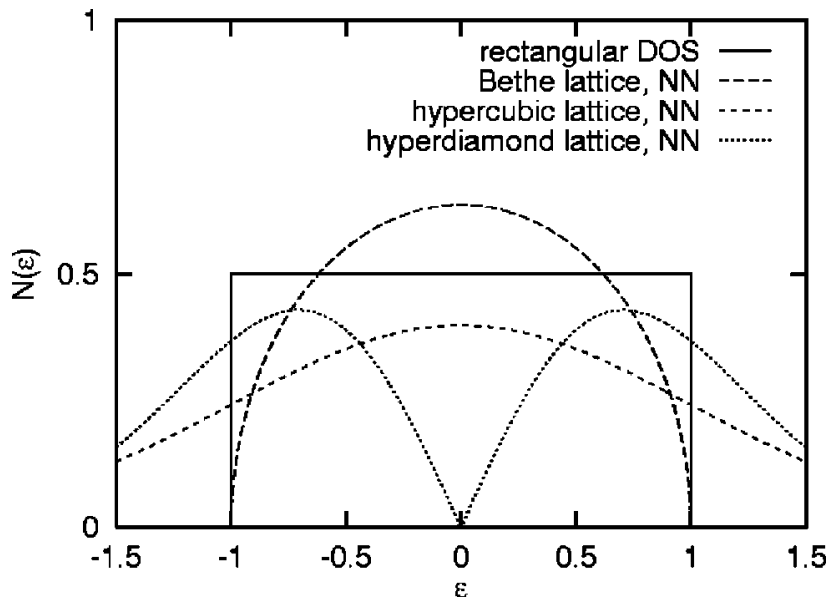

FIG. 3. DOS's for several noninteracting systems in infinite dimensions.

eral cases both methods were applied, and yielded the same results within numerical accuracy. Typical results for the functions $E^{(2)}(T)$ and $C^{(2)}(T)$ are shown over a wide temperature range in Fig. 4 for the linear chain with nearestneighbor hopping and for a constant DOS in infinite dimensions. There is a maximum at lower temperature and a minimum at higher temperature in $E^{(2)}(T)$, corresponding to the two zeros of $C^{(2)}(T)$. These are the temperatures where the specific heat curves cross for $U \rightarrow 0$.

Numerical values for $T_{+}$and $C_{+}$are listed in Table I. In view of the drastically different DOS shapes (see Figs. 2 and 3 ), it is quite remarkable that the values of $C_{+}$for these systems are very similar, ranging from 0.331 to 0.358 . For the DOS with tunable parameter $C_{+}$is plotted vs $\alpha$ in Fig. 5. Again, $C_{+}$varies only by a small amount although the shapes of the DOS change strongly. In particular, for the semimetallic DOS [Eq. (18)] $C_{+}$lies between the values for the constant and two $\delta$-peak DOS, since these are the limits of Eq. (18) for $\alpha \rightarrow 0, \infty$.

These results raise the following questions which will be addressed in Sec. V: (i) Why is $C_{+}$at the high-temperature crossing point so insensitive against changes of the DOS of

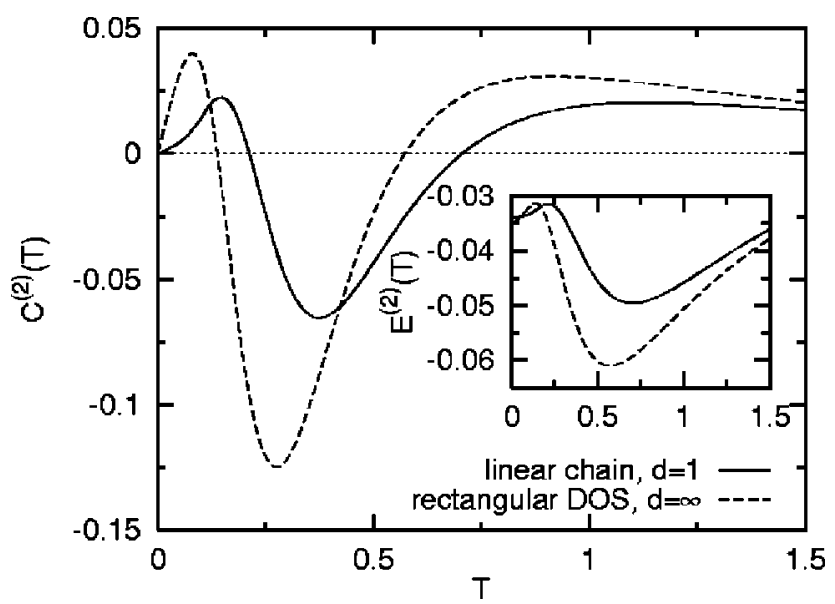

FIG. 4. Second-order contribution in $U$ to the specific heat, $C^{(2)}(T)$, for the linear chain (with NN hopping) and for the constant DOS in infinite dimensions. The half-band-width is set to 1 in both cases. The inset shows the correlation energy/ $U^{2}$.
TABLE I. Values of the crossing temperature $T_{+}$and specific heat $C_{+}$at the high-temperature crossing point for several $d$-dimensional Hubbard models. The temperature is given in units of the half-band-width, except for the hypercubic and hyperdiamond lattice, for which the variance of the DOS is set to unity.

\begin{tabular}{lcccc}
\hline \hline \multicolumn{1}{c}{ System } & Hopping & $d$ & $C_{+}$ & $T_{+}$ \\
\hline \hline linear chain & $1 / r$ & 1 & 0.346994 & 0.561816 \\
linear chain & $\mathrm{NN}$ & 1 & 0.355547 & 0.705047 \\
square lattice & $\mathrm{NN}$ & 2 & 0.352682 & 0.443585 \\
simple cubic & $\mathrm{NN}$ & 3 & 0.348327 & 0.358091 \\
body-centered-cubic & $\mathrm{NN}$ & 3 & 0.357578 & 0.241221 \\
hypercubic & $\mathrm{NN}$ & $\infty$ & 0.343630 & 0.847667 \\
hyperdiamond & $\mathrm{NN}$ & $\infty$ & 0.338411 & 0.983569 \\
Bethe lattice & $\mathrm{NN}$ & $\infty$ & 0.340906 & 0.480185 \\
rectangular DOS & undeterm. & $\infty$ & 0.339352 & 0.571895 \\
two $\delta$-peaks DOS & undeterm. & $\infty$ & 0.330857 & 1.115358 \\
\hline
\end{tabular}

the noninteracting electrons and the spatial dimension? (ii) What determines the (small) spread in the values of $C_{+}$? (iii) Why does the value of $C_{+}$at the low-temperature crossing point vary so much more strongly?

\section{EXPANSIONS OF $C_{+}$}

We will see that the influence of the DOS and of the crystal lattice on $C_{+}$can be understood by expanding around the limit of $d=\infty$. In addition, in $d=\infty$ the dispersion $\epsilon_{\mathbf{k}}$ enters only via the DOS $N(\epsilon)$, so that the effect of its form on $C_{+}$can be studied by expanding in terms of the difference between $N(\epsilon)$ and a reference $\operatorname{DOS} \bar{N}(\epsilon)$.

\section{A. Influence of the DOS in $d=\infty$}

First we consider infinite-dimensional systems with an arbitrary symmetric DOS $N(\epsilon)$ with finite bandwidth. The DOS is compared to a rectangular-shaped DOS with the same bandwidth, $\bar{N}(\epsilon)=\frac{1}{2}$ for $|\epsilon|<1$. Their difference is characterized by the quantity

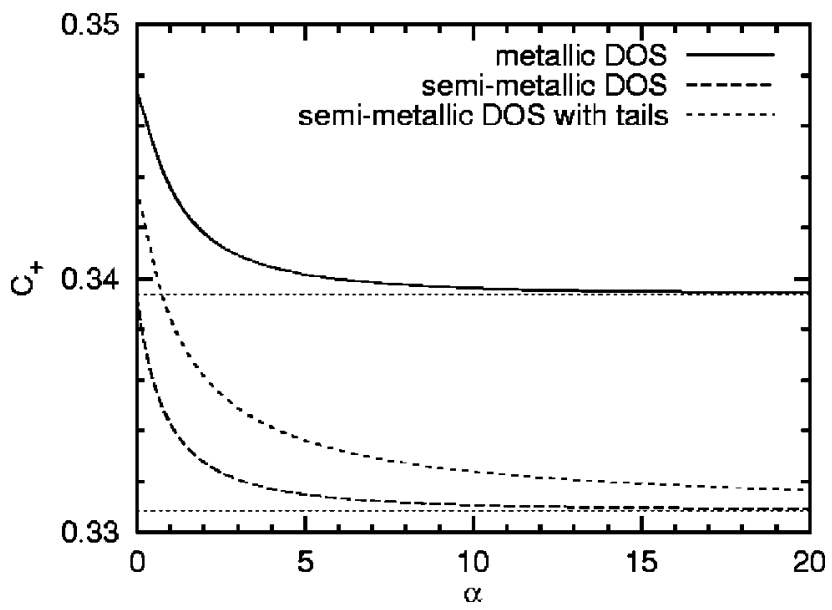

FIG. 5. $C_{+}$for several DOS's with a tunable parameter $\alpha$ [Eqs. (17)-(19)], plotted vs $\alpha$. The upper and lower dotted horizontal lines represent $C_{+}$for the constant DOS and the two $\delta$-peaks DOS, respectively. 


$$
\delta N=\int_{-1}^{1} d \epsilon|N(\epsilon)-\bar{N}(\epsilon)|
$$

which will serve as an expansion parameter. We will expand $C_{+}$to lowest order in $\delta N$, making use of the known func- tions $\bar{f}_{0}(x, \beta), \bar{C}^{(2)}(\beta)$, and $\bar{C}^{(0)}(\beta)$ pertaining to $\bar{N}(\epsilon)$, as well as the known crossing points for the rectangular DOS, $\bar{T}_{+}=1 / \bar{\beta}_{+}=0.13801$ and $\bar{C}_{+}=0.44046$ at low temperatures, while $\bar{T}_{+}=1 / \bar{\beta}_{+}=0.57190$ and $\bar{C}_{+}=0.33935$ at the high-temperature crossing point.

We begin by expanding $C^{(2)}(\beta)$ for small $\delta N$ :

$$
C^{(2)}(\beta)=\bar{C}^{(2)}(\beta)+4\left[\frac{\beta^{2}}{32} \frac{\partial^{2}}{\partial \beta^{2}} \beta^{2} \int_{0}^{1} d x\left[f_{0}(x, \beta)-\bar{f}_{0}(x, \beta)\right] \bar{f}_{0}(x, \beta)^{3}\right]+O\left[(\delta N)^{2}\right] .
$$

Using standard inequalities it can be shown that the neglected terms indeed vanish like $(\delta N)^{2}($ for fixed $\beta<\infty)$. Now $\bar{C}^{(2)}(\beta)$ is expanded around the known crossing point $\bar{\beta}_{+}$where $\bar{C}^{(2)}\left(\bar{\beta}_{+}\right)=0$. Then the new crossing point temperature is determined from the condition

$$
0 \stackrel{!}{=} C^{(2)}\left(\beta_{+}\right)=\left(\beta_{+}-\bar{\beta}_{+}\right) \bar{C}^{(2)^{\prime}}\left(\bar{\beta}_{+}\right)+4\left[\frac{\beta^{2}}{32} \frac{\partial^{2}}{\partial \beta^{2}} \beta^{2} \int_{0}^{1} d x f_{0}(x, \beta) \bar{f}_{0}(x, \beta)^{3}\right]_{\beta=\bar{\beta}_{+}}+O\left[(\delta N)^{2}\right] .
$$

To lowest order the shift in $\beta_{+}$is hence given by

$$
\delta \beta_{+}=\beta_{+}-\bar{\beta}_{+}=\int_{-1}^{1} d \epsilon N(\epsilon) \Delta \beta_{+}(\epsilon)+O\left[(\delta N)^{2}\right],
$$

where

$$
\Delta \beta_{+}(\epsilon)=-\frac{\bar{\beta}_{+}^{2}}{8 \bar{C}^{(2)^{\prime}}\left(\bar{\beta}_{+}\right)}\left[\frac{\partial^{2}}{\partial \beta^{2}} \frac{\beta^{2}}{\cosh \left(\frac{1}{2} \beta \epsilon\right)} \int_{0}^{1} d x \bar{f}_{0}(x, \beta)^{3} \cosh \left(\frac{1}{2} x \beta \epsilon\right)\right]_{\beta=\bar{\beta}_{+}} .
$$

Finally we expand $C^{(0)}(\beta)$ in $\beta-\bar{\beta}_{+}$to first order and evaluate it for $\beta=\bar{\beta}_{+}+\delta \beta_{+}$. Thus the specific heat at the crossing point $C_{+}$is obtained to lowest order in $\delta N$ as

$$
C_{+}=\bar{C}_{+}+\int_{-1}^{1} d \epsilon N(\epsilon) \Delta C_{+}(\epsilon)+O\left[(\delta N)^{2}\right]
$$

where

$$
\begin{aligned}
\Delta C_{+}(\epsilon)= & \frac{\bar{\beta}_{+}^{2} \epsilon^{2}}{2 \cosh ^{2}\left(\frac{1}{2} \bar{\beta}_{+} \epsilon\right)} \\
& +\bar{C}^{(0)^{\prime}}\left(\bar{\beta}_{+}\right) \Delta \beta_{+}(\epsilon)-\bar{C}^{(0)}\left(\bar{\beta}_{+}\right) .
\end{aligned}
$$

The last two equations show how the first order effect on $C_{+}$ of a deviation of $N(\epsilon)$ from a rectangular shape can be determined by a single integration.

The numerical evaluations of the function $\Delta C_{+}(\epsilon)$ are plotted in Fig. 6 for both crossing points. The amplitudes of the function $\Delta C_{+}(\epsilon)$ corresponding to the low- and hightemperature crossing points are seen to differ greatly, i.e., by a factor of about 40 . This implies a much greater sensitivity towards changes of the DOS and the dimension of the value of $C_{+}$at the low-temperature crossing point.

It is also clear that $C_{+}$is not entirely universal at the high-temperature crossing point, since for a general density of states $N(\epsilon)$ the integral in Eq. (25) does not vanish. We can in fact estimate the maximum value of the shift $\delta C_{+}$ $=C_{+}-\bar{C}_{+}$for arbitrary $N(\epsilon)$ with finite bandwidth, using simple integral inequalities:

$$
\left|\delta C_{+}\right| \leqslant \min \left(a_{1}, a_{2} \cdot \max _{0 \leqslant \epsilon \leqslant 1}[N(\epsilon)]\right)+O\left[(\delta N)^{2}\right]
$$

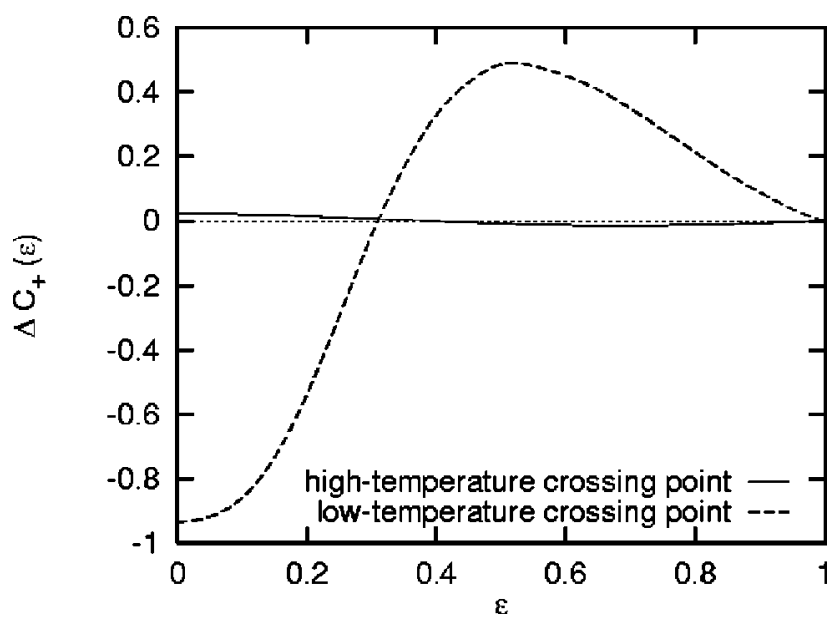

FIG. 6. Weight function $\Delta C_{+}(\epsilon)$ determining the shift in $C_{+}$at the low- and high-temperature crossing points for a Hubbard model in $d=\infty$ with finite bandwidth, according to Eq. (25). The halfband-width is set to unity. 
TABLE II. Comparison of the approximate results for $C_{+}$at the high-temperature crossing point obtained from the expansion in $\delta N$ [Eq. (25)] with the exact values for several DOS's in $d=\infty$. The expansion is controlled by the parameter $\delta N$, which measures the "distance" of $N(\epsilon)$ to the rectangular DOS, see Eq. (20). "Metallic" and "semimetallic'" refer to the DOS's of Eqs. (17) and (18), respectively. The last column shows the difference between the approximate and the exact results in percent.

\begin{tabular}{lccccc}
\hline \hline \multicolumn{1}{c}{ DOS } & $\alpha$ & $\delta N$ & $C_{+, \text {approx }}$ & $C_{+, \text {exact }}$ & diff. \\
\hline \hline rectangular & & 0 & 0.339352 & 0.339352 & 0 \\
Bethe lattice & & 0.231 & 0.341391 & 0.340906 & $0.14 \%$ \\
metallic & 4 & 0.267 & 0.341271 & 0.340444 & $0.24 \%$ \\
metallic & 6 & 0.207 & 0.340482 & 0.339966 & $0.15 \%$ \\
metallic & 8 & 0.169 & 0.340091 & 0.339743 & $0.10 \%$ \\
metallic & 10 & 0.143 & 0.339871 & 0.339621 & $0.07 \%$ \\
metallic & 12 & 0.124 & 0.339735 & 0.339552 & $0.05 \%$ \\
semimetallic & 0.1 & 0.070 & 0.338349 & 0.338381 & $0.02 \%$ \\
semimetallic & 0.5 & 0.296 & 0.335393 & 0.335880 & $0.14 \%$ \\
semimetallic & 1 & 0.500 & 0.333279 & 0.334253 & $0.29 \%$ \\
semimetallic & 2 & 0.770 & 0.331629 & 0.332752 & $0.35 \%$ \\
\hline \hline
\end{tabular}

with $a_{1} \equiv \max _{0 \leqslant \epsilon \leqslant 1}\left|\Delta C_{+}(\epsilon)\right|, a_{2} \equiv \int_{-1}^{1} d \epsilon\left|\Delta C_{+}(\epsilon)\right|$. At the high-temperature crossing point we find $a_{1}=0.02268$ and $a_{2}=0.02200$, i.e., to order $O(\delta N)$ we have $\left|\delta C_{+}\right| \ll \bar{C}_{+}$, which is the reason for the insensitivity of $C_{+}$to changes in $N(\epsilon)$. Furthermore the predicted range of values 0.339 \pm 0.023 corresponds well to the observed range of $0.331-$ 0.358; see Table I. On the other hand, at the low-temperature crossing point we find $a_{1}=0.9330$ and $a_{2}=0.7727$, so that $\left|\delta C_{+}\right| \approx \bar{C}_{+}$. Hence $C_{+}$is indeed not confined to a small interval in this case.

To check the validity of this expansion we applied Eq. (25) to several infinite-dimensional systems at the hightemperature crossing point. Results for the Bethe lattice, as well as for the metallic DOS [Eq. (17)] and the semimetallic DOS [Eq. (18)] at several values of the parameter $\alpha$ are given in Table II. As expected we find that the lowest-order approximation in Eq. (25) describes the behavior of $C_{+}$very well if the deviation from the rectangular DOS is not too large. The difference between the exact value of $C_{+}$and the approximate value $\bar{C}_{+}+\delta C_{+}$is due to corrections of order $(\delta N)^{2}$, and hence is typically an order of magnitude smaller than the first-order correction $\delta C_{+}$.

Hence we have shown that for a small change $\delta N$ in the density of states the variation of $C_{+}$at the low-temperature crossing point is large, while at the high-temperature crossing point it is small and well described by the first-order correction in $\delta N$. This gives quantitative answers to the questions posed at the end of Sec. IV.

\section{B. Lattice effects in dimension $d=1,2,3$}

To determine the influence of the lattice dimension on the value of $C_{+}$we study the hypercubic lattice with NN hopping $t$ and the scaling ${ }^{14} t=1 / \sqrt{2 d}$ and expand $C_{+}$in $1 / d$. Since the large variation of $C_{+}$at the low-temperature crossing point can already be understood from the results of Sec. $\mathrm{VA}$, we will perform this calculation only for the hightemperature crossing point.

As discussed in Sec. II, for $d=\infty$ only the local term involving $f_{0}(x, \beta)$ remains in the lattice sum in Eq. (13). The
$1 / d$ corrections are given by the contribution from the $2 d$ nearest neighbors vectors such as $\mathbf{R}_{1}=(1,0,0, \ldots){ }^{15}$

$$
\begin{aligned}
\sum_{m} f_{m}(x, \beta)^{4} & =f_{0}(x, \beta)^{4}+2 d f_{1}(x, \beta)^{4}+\cdots \\
& =f_{0}(x, \beta)^{4}+\frac{1}{2 d}\left[\frac{2}{\beta} \frac{\partial}{\partial x} f_{0}(x, \beta)\right]^{4}+O\left(d^{-2}\right),
\end{aligned}
$$

where a partial derivative with respect to $x$ was employed in the second line to remove the factor $\cos \left(\mathbf{k} \cdot \mathbf{R}_{1}\right)$ from $f_{1}(x, \beta)$. Hence the corrections to $C_{+}$in order $1 / d$ can be calculated entirely from $f_{0}(x, \beta)$. This function contains an integral over the DOS $N(\epsilon)$ only, which in turn must be expanded in $1 / d{ }^{15}$ Then the finite- $d$ correction to $T_{+}$and $C_{+}$ can be calculated by expanding Eqs. (15) and (16) to first order in $1 / d$, with the following results for the hightemperature crossing point:

$$
\begin{gathered}
C_{+}=0.343630+0.013599 \frac{1}{d}+O\left(d^{-2}\right), \\
T_{+}=\left[0.847667+0.082650 \frac{1}{d}+O\left(d^{-2}\right)\right] \frac{1}{\sqrt{2 d}} .
\end{gathered}
$$

Here the temperature scale has been reset to our previous choice of unit half-band-width in finite dimensions (i.e., the extra factor $1 / \sqrt{2 d}$ must be omitted in order to recover $T_{+}$in $d=\infty)$. Again this expansion compares very well with the numerical results; see Table III. Note that the coefficient of $d^{-1}$ in Eq. (29) is already much smaller than $C_{+}$in $d=\infty$, which is the reason for the insensitivity of $C_{+}$to lattice effects, such as Brillouin-zone shape and momentum conservation. We expect that for longer-range hopping the smallness of the deviations in the value of $C_{+}$may equally be traced to $1 / d$ corrections. 
TABLE III. Comparison of the approximate results for $C_{+}$at the high-temperature crossing point obtained from the expansion in $1 / d$ [Eq. (29)] with the exact values for hypercubic lattices in $d$ dimensions. The last column shows the difference between the approximate and the exact results in percent.

\begin{tabular}{lcccc}
\hline \hline \multicolumn{1}{c}{ Lattice } & $d$ & $C_{+, \text {approx }}$ & $C_{+, \text {exact }}$ & diff. \\
\hline \hline hypercubic & $\infty$ & 0.343630 & 0.343630 & 0 \\
simple cubic & 3 & 0.348164 & 0.348327 & $0.05 \%$ \\
square lattice & 2 & 0.350430 & 0.352682 & $0.64 \%$ \\
linear chain & 1 & 0.357229 & 0.355547 & $0.47 \%$ \\
\hline \hline
\end{tabular}

\section{CONCLUSION}

For many correlated electronic systems the curves of the specific heat vs temperature obtained for different values of a second thermodynamic parameter $X$ are known to intersect. ${ }^{1}$ For the Hubbard model at half-filling the specific heat curves for different values of the Hubbard interaction $U$ cross twice, the crossing point at high-temperatures being remarkably sharp up to intermediate values of $U$. We observed that $C_{+}$, the value of the specific heat at this crossing point in the weak-coupling limit, is practically the same for a several different lattice systems. We analyzed the origin of this conspicuous feature by calculating $C_{+}$in perturbation theory in $U$. We found the values of $C_{+}$at the high-temperature crossing point to occur in very small interval, i.e., $C_{+} \approx 0.34$ is indeed almost independent of dimensionality, crystal lattice, and energy dispersion. This is not the case for the crossing point at low temperatures, where $C_{+}$varies on a much larger scale.

Qualitatively, the reason for this difference can be traced to the relevant energy scales on which $C(T, U)$ varies. At high temperatures, the energy scale for $T$ is essentially determined by the bandwidth, i.e., by the hopping amplitude $t$ in the dispersion $\epsilon_{\mathbf{k}}$. At low temperatures, on the other hand, the generation of low-energy excitations (which are responsible for the strong enhancement of the low-temperature specific heat and $\partial C / \partial U>0)$ leads to a renormalized energy scale $t \rightarrow t_{\mathrm{eff}} \ll t$. The first maximum in $C(T, U)$ occurs at a temperature that is of the order of $t_{\text {eff }}$ (see Fig. 4). As a consequence, the first sign change in $\partial C / \partial U$ is also linked to $t_{\text {eff }}$, so that the intersection of $C(T, U)$ and $C(T, 0)$ at low temperatures does not occur at any predetermined value. In contrast, the second sign change in $\partial C / \partial U$ is determined only by energy scales that also appear in the noninteracting system, leading to a nearly universal value for the hightemperature intersection of $C(T, U)$ and $C(T, 0)$.

To gain a more quantitative understanding we identified two small parameters which determine the crossing point values $C_{+}$. The starting point for expansions in these small parameters is the limit of infinite dimensions $(d=\infty)$. (i) For $d=\infty$ the dependence of $C_{+}$on the shape of a DOS $N(\epsilon)$ with finite bandwidth is well described by the first-order correction in the parameter $\delta N=\int d \epsilon\left|N(\epsilon)-\frac{1}{2}\right|$. This parameter is a measure of the difference between $N(\epsilon)$ and a constant rectangular DOS. It turns out that at the hightemperature crossing point this correction is small for almost all DOS's, while it is large at the low-temperature crossing point. (ii) For hypercubic lattices in dimensions $1 \leqslant d<\infty$ the value of $C_{+}$may be obtained by an expansion around $d$ $=\infty$ in powers of $1 / d$. At the high-temperature crossing point the value of $C_{+}$of the $d$-dimensional system is already accurately determined by the first-order correction in $1 / d$ even for $d$ as low as $d=1$, due to the smallness of the prefactor of this term. These expansions show in detail why $C_{+}$has an almost universal value at the high-temperature crossing point.

\section{APPENDIX: INTERNAL ENERGY AT WEAK COUPLING}

The internal energy per lattice site $E$ is given by ${ }^{18}$

$$
\begin{aligned}
E(T, U)= & T \int d \mathbf{k} \sum_{\omega_{n}, \sigma} e^{i \omega_{n} \eta} \\
& \times \frac{\epsilon_{\mathbf{k}}+\frac{1}{2} \Sigma_{\sigma}\left(\mathbf{k}, i \omega_{n}\right)}{G_{0}\left(i \omega_{n}, \mathbf{k}\right)^{-1}-\Sigma_{\sigma}\left(\mathbf{k}, i \omega_{n}\right)},
\end{aligned}
$$

where $\omega_{n}$ denotes fermionic Matsubara frequencies, $\eta$ $\rightarrow 0^{+}$, and $\hbar \equiv 1$. The noninteracting Green function is $G_{0}\left(i \omega_{n}, \mathbf{k}\right)^{-1}=i \omega_{n}-\left(\epsilon_{\mathbf{k}}-\mu\right)$, and $\Sigma_{\sigma}\left(\mathbf{k}, i \omega_{n}\right)$ is the selfenergy for spin $\sigma$. In the paramagnetic phase the sum over spins just gives a factor of 2 , and the spin indices on the self-energy can be dropped.

For a symmetric DOS the chemical potential $\mu$ at halffilling is given by $U / 2$ for all temperatures due to particlehole symmetry. It is useful to define shifted functions $\hat{G}_{0}^{-1}$ $=G_{0}^{-1}-U / 2$ and $\hat{\Sigma}=\Sigma-U / 2$, with the new chemical potential fixed at 0 . Up to second order in $U \hat{\Sigma}$ is given by only one Feynman diagram where the lines now represent $\hat{G}_{0}$. We write $\hat{\Sigma}\left(\mathbf{k}, i \omega_{n}\right)=U^{2} \cdot \hat{\sigma}\left(\mathbf{k}, i \omega_{n}\right)+O\left(U^{3}\right)$, with

$$
\begin{aligned}
& \hat{\sigma}\left(\mathbf{k}_{1}, i \omega_{n}\right)=\bigcirc_{\omega_{l}, \nu_{m}} \int d \mathbf{k}_{2} \int d \mathbf{k}_{2} \int d \mathbf{k}_{3} \\
&-T^{2} \hat{G}_{0}\left(\mathbf{k}_{2}, i \omega_{l}\right) \hat{G}_{0}\left(\mathbf{k}_{3}, i \omega_{l}+i \nu_{m}\right) \hat{G}_{0}\left(\mathbf{k}_{4}, i \omega_{n}+i \nu_{m}\right) \sum_{\mathbf{K}} \delta\left(\mathbf{k}_{1}-\mathbf{k}_{2}+\mathbf{k}_{3}-\mathbf{k}_{4}-\mathbf{K}\right) .
\end{aligned}
$$

where $\nu_{m}$ denote bosonic Matsubara frequencies and the sum is over reciprocal-lattice vectors $\mathbf{K}$. Expansion of the internal energy [Eq. (A1)] in powers of $U$ yields Eqs. (7) and (8), and

$$
E^{(2)}(T)=T \int d \mathbf{k} \sum_{\omega_{n}} \frac{e^{i \omega_{n} \eta}}{i \omega_{n}-\epsilon_{\mathbf{k}}}\left[\hat{\sigma}\left(\mathbf{k}, i \omega_{n}\right)+2 \epsilon_{\mathbf{k}} \frac{\hat{\sigma}\left(\mathbf{k}, i \omega_{n}\right)}{i \omega_{n}-\epsilon_{\mathbf{k}}}\right] .
$$


The frequency summations are carried out as usual, ${ }^{18}$ with the result

$$
T \sum_{n} \frac{\hat{\sigma}\left(\mathbf{k}_{1}, i \omega_{n}\right)}{i \omega_{n}-\epsilon_{\mathbf{k}_{1}}}=-\int d \mathbf{k}_{2} \int d \mathbf{k}_{3} \int d \mathbf{k}_{4} \frac{\sinh \left[\frac{1}{2} \beta\left(\epsilon_{\mathbf{k}_{1}}-\epsilon_{\mathbf{k}_{2}}+\epsilon_{\mathbf{k}_{3}}-\epsilon_{\mathbf{k}_{4}}\right)\right]}{8\left[\epsilon_{\mathbf{k}_{1}}-\epsilon_{\mathbf{k}_{2}}+\epsilon_{\mathbf{k}_{3}}-\epsilon_{\mathbf{k}_{4}}\right] \prod_{i=1}^{4} \cosh \left(\frac{1}{2} \beta \epsilon_{\mathbf{k}_{i}}\right)} \sum_{\mathbf{K}} \delta\left(\mathbf{k}_{1}-\mathbf{k}_{2}+\mathbf{k}_{3}-\mathbf{k}_{4}-\mathbf{K}\right) .
$$

Here we eliminate the energy denominator using the identity $\sinh \left(\frac{1}{2} \beta y\right)=\frac{1}{2} \beta \int_{0}^{1} d x \cosh \left(\frac{1}{2} x \beta y\right)$. Next we rewrite the second term in square brackets in Eq. (A3) as a formal derivative $2 \epsilon_{\mathbf{k}_{1}} \partial / \partial \epsilon_{\mathbf{k}_{1}}$ of Eq. (A4). We make the integrand symmetric in all $\epsilon_{\mathbf{k}_{i}}$ by shifting $\mathbf{k}_{2}$ and $\mathbf{k}_{4}$ by $\mathbf{Q}$, where $\epsilon_{\mathbf{k}+\mathbf{Q}}=-\epsilon_{\mathbf{k}}$. Then the derivative can be replaced by $2 \beta \partial / \partial \beta$ because only products $\beta \epsilon_{\mathbf{k}_{i}}$ appear. Taking $\beta$ inside the derivative we finally arrive at

$$
\begin{aligned}
E^{(2)}(T)= & -\frac{\partial}{\partial \beta} \frac{\beta^{2}}{32} \int_{0}^{1} d x \int d \mathbf{k}_{1} \int d \mathbf{k}_{2} \int d \mathbf{k}_{3} \int d \mathbf{k}_{4} \\
& \times \frac{\cosh \left[\frac{1}{2} x \beta\left(\epsilon_{\mathbf{k}_{1}}+\epsilon_{\mathbf{k}_{2}}+\epsilon_{\mathbf{k}_{3}}+\epsilon_{\mathbf{k}_{4}}\right)\right]}{\cosh \left(\frac{1}{2} \beta \epsilon_{\mathbf{k}_{1}}\right) \cosh \left(\frac{1}{2} \beta \epsilon_{\mathbf{k}_{2}}\right) \cosh \left(\frac{1}{2} \beta \epsilon_{\mathbf{k}_{3}}\right) \cosh \left(\frac{1}{2} \beta \epsilon_{\mathbf{k}_{4}}\right)} \sum_{\mathbf{K}} \delta\left(\mathbf{k}_{1}-\mathbf{k}_{2}+\mathbf{k}_{3}-\mathbf{k}_{4}-\mathbf{K}\right) .
\end{aligned}
$$

In $d=1,2$, and 3 this simplifies to Eq. (9), while for $d=\infty$ the $\delta$ function can be omitted ${ }^{15}$ and the numerator can be replaced by $\Pi_{i=1}^{4} \cosh \left(\frac{1}{2} x \beta \epsilon_{i}\right)$, leading to Eq. (10).

The integrals in Eq. (A5) also factorize if we express momentum conservation as a sum over lattice vectors $\mathbf{R}_{m}$,

$$
\sum_{\mathbf{K}} \delta\left(\mathbf{k}_{1}-\mathbf{k}_{2}+\mathbf{k}_{3}-\mathbf{k}_{4}-\mathbf{K}\right)=\sum_{m} \exp \left[i\left(\mathbf{k}_{1}-\mathbf{k}_{2}+\mathbf{k}_{3}-\mathbf{k}_{4}-\mathbf{K}\right) \cdot \mathbf{R}_{m}\right]
$$

leading to Eqs. (13) and (14). Since the functions $f_{m}(x, \beta)$ in Eq. (14) cannot be calculated in closed form for general $\epsilon_{\mathbf{k}}$ we employ a high-temperature expansion, which yields

$$
\begin{gathered}
f_{m}(x, \beta)=\sum_{n=0}^{\infty} \frac{\beta^{n}}{n !} E_{n}\left(\frac{1+x}{2}\right) I_{n}\left(\mathbf{R}_{m}\right), \\
I_{n}\left(\mathbf{R}_{m}\right)=\int d \mathbf{k}\left(\epsilon_{\mathbf{k}}\right)^{n} \exp \left(i \mathbf{k} \cdot \mathbf{R}_{m}\right),
\end{gathered}
$$

where $E_{n}(x)$ are Euler polynomials. The remaining Brillouin-zone integrals are calculated as follows. For the $d$-dimensional hypercubic lattice the lattice vectors are $\mathbf{R}_{m}=\left(m_{1}, \ldots m_{d}\right)$ with integer $m_{i}$, so that for NN hopping $t$ we have

$$
I_{n}^{\mathrm{hc}}\left(\mathbf{R}_{m}\right)=(-t)^{n} \sum_{n_{1}+\cdots n_{d}=n}\left(\begin{array}{c}
n \\
n_{1}, \ldots n_{d}
\end{array}\right) \prod_{i=1}^{d}\left[\int_{-\pi}^{\pi} \frac{d k_{i}}{2 \pi}\left(2 \cos k_{i}\right)^{n_{i}} \cos \left(k_{i}\left|m_{i}\right|\right)\right] .
$$

The integral in square brackets equals $\left(\begin{array}{c}n_{i} \\ r_{i}\end{array}\right)$ if $0 \leqslant n_{i}-\left|m_{i}\right|=2 r_{i}$ with integer $r_{i}$, and zero otherwise. Hence

$$
I_{n}^{\mathrm{hc}}\left(\mathbf{R}_{m}\right)=(-t)^{n} n ! \sum_{r_{1}, \ldots, r_{d}}{ }^{\prime} \prod_{i=1}^{d} \frac{1}{r_{i} !\left(r_{i}+\left|m_{i}\right|\right) !}
$$

where the sum is restricted to $2 r_{1}+\cdots+2 r_{d}=n-M^{\mathrm{hc}}$, with $M^{\mathrm{hc}} \equiv \sum_{i=1}^{d}\left|m_{i}\right|$. Note that $I_{n}^{\mathrm{hc}}$ vanishes if $M^{\mathrm{hc}}>n$ or if $n$ $+M^{\text {hc }}$ is odd. For the bcc lattice in $d=3$ we use the hypercubic lattice basis $\mathbf{R}_{m}$ and the dispersion given in Sec. III. We obtain

$$
I_{n}^{\mathrm{bcc}}\left(\mathbf{R}_{m}\right)=(-t)^{n} \prod_{i=1}^{3}\left[\int_{-\pi}^{\pi} \frac{d k_{i}}{2 \pi}\left(2 \cos k_{i}\right)^{n} \cos \left(k_{i}\left|m_{i}\right|\right)\right]=(-t)^{n} \prod_{i=1}^{3}\left(\begin{array}{l}
n \\
r_{i}
\end{array}\right),
$$

with $r_{i}$ defined as above. In particular $I_{n}^{\mathrm{bcc}}$ vanishes if $M^{\mathrm{bcc}} \equiv \max _{i}\left|m_{i}\right|>n$ or if $n+\left|m_{i}\right|$ is odd. The advantage of the present high-temperature expansion is that terms with $M>n$ vanish when expanding to order $\beta^{n}$, so that the lattice sum terminates at $m_{i}=n$. In addition, lattice symmetries can be used to further reduce the computational effort. This makes the calculation feasible even in dimension $d=3$, where numerical Brillouin-zone integration is usually difficult. At the high-temperature crossing point $O\left(\beta^{80}\right)$ is typically sufficient to obtain $T_{+}$and $C_{+}$to an accuracy of $10^{-8}$. 
*Present address: Sektion Physik, Ludwig-Maximilians-Universität München, Theresienstr. 37, 80333 München, Germany.

${ }^{1}$ D. Vollhardt, Phys. Rev. Lett. 78, 1307 (1997).

${ }^{2}$ D. F. Brewer, J. G. Daunt, and A. K. Sreedhar, Phys. Rev. 115, 836 (1959); D. S. Greywall, Phys. Rev. B 27, 2747 (1983).

${ }^{3}$ G. E. Brodale, R. A. Fisher, N. E. Phillips, and J. Flouquet, Phys. Rev. Lett. 56, 390 (1986).

${ }^{4}$ N. E. Phillips, R. A. Fisher, J. Flouquet, A. L. Giorgi, J. A. Olsen, and G. R. Stewart, J. Magn. Magn. Mater. 63\&64, 332 (1987).

${ }^{5}$ H. G. Schlager, A. Schröder, M. Welsch, and H. v. Löhneysen, J. Low Temp. Phys. 90, 181 (1993).

${ }^{6}$ T. Brugger, T. Schreiner, G. Roth, and G. Czjzek, Phys. Rev. Lett. 71, 2481 (1993).

${ }^{7}$ J. Hubbard, Proc. R. Soc. London, Ser. A 276, 238 (1963); M. C. Gutzwiller, Phys. Rev. Lett. 10, 159 (1963); J. Kanamori, Prog. Theor. Phys. 30, 275 (1963).

${ }^{8}$ H. Shiba and P. A. Pincus, Phys. Rev. B 5, 1966 (1972); H. Shiba, Prog. Theor. Phys. 48, 2171 (1972).

${ }^{9}$ G. Jüttner, A. Klümper, and J. Suzuki, Nucl. Phys. B 522, 471 (1998).
${ }^{10}$ D. Duffy and A. Moreo, Phys. Rev. B 55, 12918 (1997); ibid. 56, 7022(E) (1997).

${ }^{11}$ A. Georges and W. Krauth, Phys. Rev. B 48, 7167 (1993).

${ }^{12}$ F. Gebhard and E. Ruckenstein, Phys. Rev. Lett. 68, 244 (1991); F. Gebhard, A. Girndt, and A. Ruckenstein, Phys. Rev. B 49, 10 926 (1994).

${ }^{13}$ T. Usuki, N. Kawakami, and A. Okiji, J. Phys. Soc. Jpn. 59, 1357 (1990).

${ }^{14}$ W. Metzner and D. Vollhardt, Phys. Rev. Lett. 62, 324 (1989). For reviews, see D. Vollhardt, in Correlated Electron Systems, edited by V. J. Emery (World Scientific, Singapore, 1993), p. 57; A. Georges, G. Kotliar, W. Krauth, and M. Rozenberg, Rev. Mod. Phys. 68, 13 (1996).

${ }^{15}$ E. Müller-Hartmann, Z. Phys. B 74, 507 (1989).

${ }^{16}$ G. Santoro, M. Airoldi, S. Sorella, and E. Tosatti, Phys. Rev. B 47, 16216 (1993).

${ }^{17}$ W. H. Press, S. A. Teukolsky, W. T. Vetterling, and B. P. Flannery, Numerical Recipes in $C$ (Cambridge University Press, Cambridge, 1992), and references therein.

${ }^{18}$ A. L. Fetter and J. D. Walecka, Quantum Theory of ManyParticle Systems (McGraw-Hill, New York, 1971). 